\title{
G

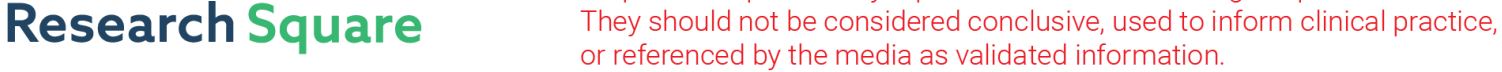 \\ The hematopoietic function of medicinal wine Maoji Jiu revealed in blood deficiency model rats
}

\section{Yongli Xu}

Guangxi Botanical Garden of Medicinal Plants

\section{Fanqiang Zeng}

Department of Pharmacy, Guigang City People's Hospital, The Eighth Affiliated Hospital of Guangxi Medical University

\section{Jianping Jiang}

Guangxi Botanical Garden of Medicinal Plants

\section{Juan Huo}

Guangxi Botanical Garden of Medicinal Plants

\section{Chengjian Zhao}

Guangxi Botanical Garden of Medicinal Plants

\section{Zhigang Yan (D Izg7898@163.com)}

Guangxi Botanical Garden of Medicinal Plants

\section{Li Li}

Guangxi Institute of Chinese Medicine \& Pharmaceutical Science

\section{Research Article}

Keywords: Maoji Jiu, blood deficiency, hematopoietic function, hematopoietic growth factor

Posted Date: January 21st, 2022

DOI: https://doi.org/10.21203/rs.3.rs-1213323/v1

License: (c) (1) This work is licensed under a Creative Commons Attribution 4.0 International License. Read Full License 


\section{Abstract}

Background: Maoji Jiu (MJ), a medicinal wine, has been used commonly by Chinese to enrich and nourish the blood. In this study, the aim is to examine the hematopoietic function of $\mathrm{MJ}$ and investigate its anti-blood deficiency mechanism.

Methods: 36 rats were randomly divided into six groups with six rats in each group. The blood deficiency model was induced by injecting with $\mathrm{N}$-acetylphenylhydrazine (APH) and injected intraperitoneally with cyclophosphamide (CTX), and treatment drugs were given by oral gavage twice each day for continuous 10 days from the start of experiments.

Results: The administration of MJ improved the levels of white blood cells (WBC), red blood cells (RBC), hemoglobin (HGB) and hematocrit (HCT) in the blood deficiency model rats. Hematopoietic effect involves in regulating the antioxidant activity in the liver and the levels of Bcl-2, Bax, erythropoietin (EPO), transforming growth factor-beta-1 (TGF- $\beta 1$ ) and macrophage colony-stimulating factor (M-CSF) mRNA in spleen tissues to enhance extramedullary hematopoiesis.

Conclusion: This study suggests that MJ has a beneficial effect on blood deficiency model rats.

\section{Background}

Anemia, the most common disease of blood, is a condition associated with an insufficient number of healthy red blood cells resulting in lower hemoglobin levels. It is often induced by increased erythrocyte damage, decreased or faulty RBC production, and massive blood loss. ${ }^{1}$ Physiological conditions, such as menstruation, pregnancy and postpartum, occur in the female and therefore the anemic syndrome is more common in women than men and widely affects women's health. In addition, malignant tumor chemotherapy also has a common adverse reaction on hematopoietic function, which can exhibit leucopoenia and thrombocytopenia in patients. ${ }^{2}$ This is a major adverse effect when treating malignant tumor patients by using chemotherapy. Therefore, improving hematopoietic function in anemic patients and preventing the side-effects of malignant tumor chemotherapy is a major goal of research in this field of medicine. In the recent years, it has been reported that many traditional Chinese medicines (TCMs) could treat blood deficiency because of their unique effect and reduced toxicity. ${ }^{3,4}$

The blood deficiency model is usually induced by $\mathrm{N}$-acetyl phenylhydrazine (APH) and Cyclophosphamide (CTX) in previous studies. ${ }^{5,6} \mathrm{APH}$ is a strong oxidizing agent that decreases the production of glutathione and destroys the stability of red blood cell membranes. ${ }^{7}$ CTX is an anti-tumor drug commonly used in clinic, which has a primary adverse effect on the immune organs and the peripheral routine blood. ${ }^{8}$ Therefore, the curative effect evaluation of hemopoietics can be from peripheral routine blood, antioxidant capacity and immune organs. Previous study showed that haemopoiesis is the process by which haematopoietic stem cells develop into mature blood cells through 
the stimulation of various cytokines in the haematopoietic microenvironment, including EPO, TGF- $\beta_{1}$, and M-CSF. ${ }^{9}$

Maoji Jiu (MJ) is a Chinese health-care wine used commonly for treating women's ailments. It has notable effects on nourishing the blood and regulating menstruation and these were recorded in "Guangxi Zhong Yao Zhi" (published in 1959) and "Quan Guo Zhong Cao Yao Hui Bian" (published in 2014). It consists of Centropus sinensis (Hong-mao-ji or He-chi-ya-juan), Angelica sinensis Radix (Danggui), Chuangxiong Rhizoma (Chuanxiong), Angelicae dahuricae Radix (Baizhi), Carthami Flos (Honghua), Homalomenae Rhizoma (Qian-nian-jian), Poria cocos (Fuling), Paeoniae lactiflora Pall Radix (Chishao), Semen persicae (Taoren) and some wine in the ratios of 32: 32: 32: 32: 32: 32:4: $3: 3$, respectively, with a final ratio of medical materials to wine of 1:17. The beneficial hematopoietic effect of the components of these TCMs has been proved in several previous studies. ${ }^{5,6,10-13}$ Dating back to the late Qing Dynasty and the early Republic of China, MJ was first used in Guangdong and Guangxi provinces, and in the next few decades has been used extensively all over the world. Recently, MJ has a well-deserved reputation in China and Southeast Asian Nations because of its notable quality and efficacy.

Although MJ has been used commonly and widely for the treatment of blood deficiency for many years, there is no any scientific study, to our best knowledge, on its potential pharmacological property.

Therefore, it is necessary to confirm the hematopoietic function of $\mathrm{MJ}$ and further explore its mechanism using a scientific approach, which will provide the basis for its clinical application. In this study, blood deficiency model rat is used to investigate the mechanism of hematopoietic function of MJ. To evaluate the hematopoietic activity of MJ, Fufang Ejiao Jiang (FEJ), a drug has been shown to possess powerful hematopoietic effects, ${ }^{14}$ was used as the positive control drug in this study.

\section{Materials And Methods}

\section{Chemicals and reagents}

$\mathrm{N}$-acetyl phenylhydrazine (APH) was purchased from Shanghai Ziyi Reagent Factory (Shanghai, China, No. ZY150623). Cyclophosphamide (CTX) was purchased from Baxter Oncology GmbH (Germany, No. 5J078A). The superoxide dismutase (SOD), total antioxidant activity (T-AOC) and malondialdehyde (MDA) kits were purchased from Nanjing Jiancheng Bioengineering Research Institute (Nanjing, China). Erythropoietin (EPO) ELISA kit was obtained from Wuhan Boster Bio-engineering Co. Ltd. (Wuhan, China). Medical materials and extraction protocol

Centropus sinensis was obtained from Pingnan, Guigang (Guangxi, China), and Angelica sinensis Radix, Chuangxiong Rhizoma, Angelicae dahuricae Radix, Carthami Flos, Homalomenae Rhizoma, Poria cocos, Paeoniae lactiflora Pall Radix, and Semen persicae were purchased from Yancheng Buyi Pharmacy Co. Ltd. (Jiangsu, China). All of them were identified by Dr. Li (Guangxi Botanical Garden of Medicinal Plants, Guangxi, China). The voucher specimens were deposited in Guangxi Botanical Garden of Medicinal Plants. In this study, we prepared MJ in a traditional and classical way according to a folklore practice. 
Centropus sinensis was sacrificed; the viscera were wiped off, and then the whole body was dried in an oven. After steaming with a boiler for 15 minutes, Centropus sinensis $(160 \mathrm{~g})$ was placed in a glass jar and appropriate wine (the ethanol content was 40 percent) was poured into the jar (inundated with Centropus sinensis), and then the jar was sealed for 25 days. The rest of the medical materials, Radix Angelica sinensis (160g), Radix Ligusticum chuangxiong Hort (160g), Radix Angelicae dahuricae (160g), Flos Carthami (160g), Rhizoma Homalomenae (160), Poria cocos (20g), Radix Paeoniae lactiflora Pall $(15 \mathrm{~g})$, Semen persicae $(15 \mathrm{~g})$ and the remaining wine, with the total volume up to16800 $\mathrm{ml}$, was added into the same jar. The jar was sealed again for additional 55 days and then filtered through gauze. The accumulated filtrate was evaporated with a rotary evaporator under vacuum at $50^{\circ} \mathrm{C}$. The ethanol content of the concentrate was adjusted to 40 percent $(\mathrm{v} / \mathrm{v})$, and the total volume was $8400 \mathrm{ml}$. The wine was stored at room temperature till further use.

\section{Animals}

Female Sprague-Dawley rats, with weight between 180 and 220g, were provided by Guangxi Medical University Laboratory Animal Centre (Nanning, China, License No. SCXK [Gui] 2014-0002). All rats were kept in a case at a temperature of $20-25^{\circ} \mathrm{C}$ and a relative humidity of $40-60 \%$ under a 12 -h light/dark cycle with free access to tap water and standard diet. Rats were allowed to acclimatize for five days before the experiment. All procedures were carried in accordance with the ARRIVE (Animal Research: Reporting In Vivo Experiments) guidelines and approved by Ethics Committee of Guangxi Botanical Garden of Medicinal Plants (No. 20170301).

\section{Blood deficiency model construction and administration}

After acclimatization, the rats were randomly divided into six groups, each with six rats. The groups are control group (CG), model group (MG), FEJ group, high dose group of $M J(M J-H)$, middle dose group of MJ (MJ-M) and low dose group of MJ (MJ-L). The rats in model group, FEJ, MJ-H, MJ-M and MJ-L groups were hypodermically injected with $2 \%$ APH saline solution on the 1 st and 4 th day at the dose of $20 \mathrm{mg} / \mathrm{kg}$ and $10 \mathrm{mg} / \mathrm{kg}$, respectively; 2 hours after injections on the 4th day, the rats were intraperitoneally injected with CTX saline solution at a dose of $15 \mathrm{mg} / \mathrm{kg}$ for continuous four days. The rats in the control group were hypodermically or intraperitoneally injected with an equal volume of normal saline (NS). Thus, the blood deficiency model was created. The rats in MJ-H, MJ-M and MJ-L groups were intragastrically given $10 \mathrm{ml} / \mathrm{kg}, 5 \mathrm{ml} / \mathrm{kg}$ and $2.5 \mathrm{ml} / \mathrm{kg}$ body mass of $\mathrm{MJ}$, respectively, twice a day for 10 continuous days. The rats in FEJ group were intragastrically given $6 \mathrm{ml} / \mathrm{kg}$ body mass of FEJ twice a day for 10 continuous days. Simultaneously, rats in the control group and model group were given an equal volume of NS by oral gavage. All rats were sacrificed on the 11th day. Blood, thymus, spleen, liver and lung samples were obtained for further examination.

\section{Blood routine examination and visceral index calculation}

The blood samples of inner canthus $0.5 \mathrm{ml}$ were collected to assay the routine blood tests using a SysmexXT-2000iv full-automatic blood cell analyzer (Sysmex Company, Japan), which included white blood cell (WBC), red blood cell (RBC), hemoglobin (HGB), hematocrit (HCT) and platelets (PLT) levels. 
The visceral index, including the thymus, spleen, liver and lung, was calculated according to the formula: visceral index $=$ visceral weight $(\mathrm{mg}) /$ rat weight $(\mathrm{g})$.

\section{Assessment of antioxidant activity and lipid peroxidation}

The antioxidant activities commonly include measurements of SOD and T-AOC detected by using commercially available kits. In brief, liver tissues were homogenized on ice with NS (10\% homogenate). Then the homogenates were centrifuged at $2500 \times \mathrm{g}$ for $10 \mathrm{~min}$ at $4{ }^{\circ} \mathrm{C}$ to obtain the supernatants, which were analyzed for antioxidant activities and lipid peroxidation. The activities of SOD and T-AOC were measured by using commercially available kits according to the instructions of the manufacturer. The content of lipid peroxidation was detected by measurement of MDA formation employing a commercially available kit.

\section{Histopathological examination}

The spleen tissue was obtained and a piece was immediately fixed in phosphate buffered saline containing $4 \%$ formaldehyde. After fixation, the sample was embedded in paraffin, sectioned in $5 \mu \mathrm{m}$ thick and mounted on slides. The paraffin-embedded sections were rehydrated using xylene and alcohol series, then stained with hematoxylin-eosin (H\&E) to observe histological changes and these evaluated by using optical microscopy (Olympus, China).

\section{Immunohistochemistry}

The paraffin-embedded sections of spleen samples were also rehydrated using xylene and alcohol series, then labeled separately with rat monoclonal antibody Bcl-2 (1:100, Bioss, Beijing, China) and Bax (1:1000, Santa Cruz, China) as the primary antibodies and streptavidin-peroxidase as a secondary antibody respectively to observe the presence of these antigens under optical microscopy (Olympus, China), and Image-pro-plus 6.0 was used to analyze the Bcl-2 and Bax expressions semi-quantitative.

\section{Cytokine secretion}

The spleen tissues together with 10 times NS were homogenized using a homogenizer (Ningbo Scientz Biotechnology Co., Ltd., Ningbo, China), and then the total proteins of the homogenates were detected using a nucleic acid detector (Thermo, America). Erythropoietin (EPO) concentration in the homogenates was assayed by using the avidin-biotin peroxidase complex Enzyme-Linked Immunosorbent Assay (ABCELISA) kits according to the manufacturer's instructions.

\section{Real-time PCR}

Total spleen RNA was extracted from frozen tissue samples using Trizol reagent according to the instructions of the manufacturer. Complementary DNA (CDNA) was synthesized using $1 \mu \mathrm{g}$ of total RNA and a SuperScript ${ }^{\circledR} \otimes$ Reverse Transcriptase Kit (Takara, China). RT-PCR amplification was performed on a 7300 real time PCR detection system (Applied Biosystems, Foster City, CA, USA) and SYBR® Green PCR Master Mix (Applied Biosystems). Sample cDNAs (equivalent to $2 \mu \mathrm{g}$ of total RNA) were regarded as 
templates with gene-specific primers. The PCR primer sequences were set as follows: TGF- $\beta_{1}: 5^{\prime}$ CATTGCTGTCCCGTGCAGA-3' (forward) and 5'-AGGTAACGCCAGGAATTGTTGCTA-3'(reverse); M-CSF: 5'GAATACTGAAC-CTGCCT GCTGAA-3'(forward) and 5'-AGGCCAGCTCAGTGCAAGAA-3' (reverse); $\beta$-actin: 5'GGAGATTACTGCCCTGGCTCCTA-3' (forward) and 5'-GACTCATCGTACTCCT-

GCTTGCTG-3' (reverse). $\beta$-actin was used as the housekeeping gene. The expression levels of target mRNAs were normalized to $\beta$-actin in arbitrary units.

\section{Statistical analysis}

Statistical analysis was performed by using SPSS 16.0 and the results are expressed as mean \pm SD, where SD represents standard deviation. Statistical differences between groups were determined using a one-way analysis of variance. A value of $p<0.05$ was considered to be statistically significant.

\section{Results}

\section{Appearance and histopathological examination of the blood deficiency model rats}

The appearances of rats were changed after given APH and CTX. As showed in Fig. 1, the rats in the control group appeared to be in a good condition; their ears, noses, faces and tails were pink, and their hairs were tight and glossy. However, the rats in the model group appeared to be in a bad condition; their hairs were fluffy and shed easily, and their ears, noses, faces and feet were pale. Especially the ears of the model group were easily observed. After administration, the above features of the rats in the FEJ, MJ-H, MJ-M and MJ-L groups were similar to those in the control group. Among those, the $\mathrm{MJ}-\mathrm{H}$ was the closest.

Histopathological changes of spleen sections were observed and evaluated by HE staining (Fig. 2). The spleen tissues in the rats of the control group showed a normal spleen architecture clearly with regular distribution of red pulps (RP) and white pulps (WP), tight arrangement of lymphocytes and no obvious existence of segmentation and differentiation. In contrast, the structure of white pulps in splenocytes of the model group were destroyed. Crypta (CR) was increased and lymphocytes were decreased. In the FEJ $(6 \mathrm{ml} / \mathrm{kg})$ treatment group, the rats appeared to be better, with less connective tissue and more lymphocytes. These characteristics of rats in the model group were also observed after treatment with $\mathrm{MJ}(10 \mathrm{ml} / \mathrm{kg}, 5 \mathrm{ml} / \mathrm{kg}$ or $2.5 \mathrm{ml} / \mathrm{kg})$, but they were significantly improved compared with those in the model group.

\section{Effects of MJ on peripheral routine blood and visceral index of the blood deficiency model rats}


The indexes of peripheral routine blood directly reflect the curative effect of replenishing blood. As showed in Table 1, the WBC, RBC, HGB, HCT and PLT levels in the model group were significantly decreased compared with those in the animals in the control group $(p<0.01)$, suggesting the model was copied successfully. The WBC, RBC, HGB and HCT levels in MJ and FEJ treatment groups showed an obvious increasing trend compared with those in the animals in the model group.

The changes of visceral indexes can be reflected injure and recover of organs in a certain extent. As showed in Table 2, the liver index, thymus index and spleen index in rats in the model group were reduced significantly compared with those in the control group $(p<0.01)$. Compared with the model group, the liver index and spleen index in MJ-treated groups and FEJ showed a decreasing trend and the thymus index in the MJ and FEJ treatment groups showed an obvious increasing trend. The lung index in the control and treatment groups had no statistical difference compared with the animals in the model group.

\section{Effects of MJ on antioxidant activity and lipid peroxidation of the blood deficiency model rats}

The indicators of antioxidant capacity and lipid peroxidation mainly include SOD, T-AOC and MDA. As showed in Table 3, the activity of T-AOC presented a significant increase in rats in the control group and the blood deficiency model rats treated with MJ or FEJ compared with the animals in the model group ( $p$ $<0.05)$. In addition, the level of MDA in the animals in the model group was significantly decreased compared with those in the control group $(p<0.01)$. Compared with model group, the levels of MDA in the animals in the MJ-H, MJ-M and FEJ groups were reduced significantly $(p<0.05)$, but in the MJ-L group there was little impact on this parameter. The activity of SOD in the rats in the model group showed a little decrease compared with the other groups, but this was not statistically significant.

\section{Effects of $\mathrm{MJ}$ on apoptosis regulatory proteins and hematopoietic growth factors in spleen tissues of blood deficiency model rats}

$\mathrm{Bcl}-2$ and Bax are two proteins that play important roles in regulating cell apoptosis. In this study, we found that the expression levels of Bcl-2 and Bax, and the Bcl-2/Bax ratio decreased significantly $(p<$ $0.05)$ in blood deficiency model rats that was induced by APH and CTX. After the administration of MJ and FEJ, Bcl-2 expression level and the Bcl-2/Bax ratio of the animals in the FEJ, MJ-H and MJ-M groups increased significantly $(p<0.05)$; however Bax expression level of FEJ and $\mathrm{MJ}-\mathrm{H}$ groups increased significantly $(p<0.05)$ compared with that in the model group (Fig. 3$)$.

In present study, we detected the EPO level in spleen tissues of blood deficiency model rats, and the results are showed in Fig. 4. The EPO level in the model group was decreased significantly $(p<0.01)$ compared with in the control group. However, the EPO level of the FEJ and MJ-H groups was increased 
significantly $(p<0.05)$, and the EPO level of MJ-M and MJ-L groups was also shown an increasing trend compared with in the model group.

M-CSF and TGF- $\beta_{1}$ are two important cytokines in promoting hematopoiesis. Here, we determined and compared the M-CSF and TGF- $\beta_{1}$ mRNA levels in spleen tissues of blood deficiency model rats (Fig. 5). Compared with the control group, the M-CSF mRNA level in the other groups was decreased significantly $(p<0.05)$, but the TGF- $\beta_{1}$ mRNA level in the model group was increased significantly $(p<0.05)$. Compared with animals in the model group, the M-CSF mRNA level in FEJ and MJ-H groups was increased significantly $(p<0.05)$, but the TGF- $\beta_{1}$ mRNA level in FEJ and MJ-H groups was decreased significantly $(p<0.05)$. These results demonstrated that $\mathrm{MJ}$ could regulate hematopoiesis via M-CSF and TFG- $\beta_{1}$, with similar effect as FEJ if MJ dose is high.

\section{Discussion}

The aim of this study was to evaluate the hematopoietic function of $\mathrm{MJ}$ and investigate its mechanism. $\mathrm{APH}$, as a strong oxidizing agent, decreases the production of glutathione and destroys the stability of red blood cell membranes. ${ }^{7}$ CTX, as an anti-tumor drug commonly used in clinic, has a primary adverse effect on immune organs and the total number of reduced RBC, WBC and PLT in peripheral blood. ${ }^{8}$ Therefore, we induced rats into a blood deficiency model by hypodermically injecting with APH and intraperitoneally injecting with CTX. We detected peripheral routine blood parameters of blood deficiency model rats using a fully automated blood cell analyzer. The results shown that the rats given $\mathrm{MJ}-\mathrm{H}$ and MJ-M of MJ had significantly higher WBC, RBC, HGB and HCT levels than the animals in the model group $(p<0.05)$, which suggests that MJ could improve blood deficiency status of rats.

Having confirmed that $\mathrm{MJ}$ could improve hematopoietic function, we investigated its mechanism further. Blood deficiency syndrome may be connected with the damages of non-oxidation function. ${ }^{15,16}$ The activities of SOD and T-AOC, and the level of MDA are commonly detected to assess the anti-oxidative property in previous studies. ${ }^{8,17,18}$ In this study, the results show that the activity of T-AOC in animals in the MJ-H, MJ-M and MJ-L groups was significantly higher than those in the model group $(p<0.01)$, and the level of MDA in MJ-H and MJ-M groups was lower than the rats in the model group $(p<0.05)$ (Table $3)$. The results suggested that MJ could improve the anti-oxidative property via SOD, T-AOC and MDA in blood deficiency model rats.

$\mathrm{Bcl}-2$ and Bax gene are two apoptosis regulatory proteins that belong to the Bcl-2 gene family, which plays an important role in regulating cell apoptosis. Previous studies shown that cell viability can be monitored by measuring the ratio of Bcl-2 and Bax after activation of apoptosis. ${ }^{19,20}$ In this study, injury and apoptosis of spleen cells in the model group were observed, which were induced by APH and CTX. We also observed that when FEJ or MJ was administered, this phenomenon in the treatment groups was alleviated when compared to the model group. To better elucidate the cause behind the injury and apoptosis, we used immunohistochemical methods to determine the $\mathrm{Bcl}-2$ and $\mathrm{Bax}$ expression levels in 
spleen. Compared with the model group, the Bcl-2 and Bax expression levels and the value of $\mathrm{Bcl}-2 / \mathrm{Bax}$ ratio in the FEJ, MJ-H and MJ-M groups were significantly increased $(p<0.05)$. These results indicate that $\mathrm{MJ}$ may reduce spleen cells injury and apoptosis by regulating $\mathrm{Bcl}-2$ and $\mathrm{Bax}$ expression.

Hematopoietic growth factors from spleen cells, bone-marrow stromal cells and other cells ${ }^{21}$ play an important role in the growth and differentiation of various blood cells. ${ }^{22}$ EPO and M-SCF are two essential HGFs that participate in hematopoietic regulation. ${ }^{5,23}$ EPO regulates the erythropoiesis production and stimulates the proliferation of early erythroid precursors and the differentiation of late erythroid precursors. ${ }^{24}$ TGF- $\beta_{1}$, a member of TGF- $\beta$, plays an important role in regulating hematopoiesis. Hematopoiesis could be inhibited when it is at a high level. In the present study, the M-CSF mRNA level in the model group, FEJ, MJ-H MJ-M and MJ-L groups were higher than in animals in the control group ( $p<$ 0.01 ) and this may be connected with a self-healing mechanism when rats are in a blood deficiency status. However, the M-CSF mRNA level in the FEJ and MJ-H groups were higher than in animals in the model group $(p<0.05)$. Similarly, the EPO level in the control group, FEJ and MJ-H groups were higher than in the model group of animals $(p<0.05)$. However, the TGF- $\beta_{1}$ mRNA level in the control group, FEJ and $\mathrm{MJ}-\mathrm{H}$ groups were lower than in the model group $(p<0.05)$. These results indicate that MJ may increase hematopoietic activity by increasing the EPO and M-CSF mRNA levels and decreasing the TGF$\beta_{1}$ mRNA.

\section{Conclusions}

In this study, we have shown that $\mathrm{MJ}$, a traditional Chinese health-care wine, has hematopoietic function in vivo in a rat model. The findings indicated that $\mathrm{MJ}$ administered at high and middle dose groups significantly increased the level of WBC, RBC, HGB and HCT $(p<0.05)$, which confirms its hematopoietic effect. In this study, we observed that $\mathrm{MJ}$ can significantly increase antioxidant capacity of liver, Bcl2/Bax ratio, the levels of EPO and M-CSF mRNA of spleen, and significantly decrease the level of TGF- $\beta_{1}$ mRNA in spleen. Therefore, the hematopoietic effect of MJ may be connected with antioxidant ability, apoptosis regulatory proteins and hematopoietic growth factors. It is of interesting to confirm the mechanism of hematopoietic function of $\mathrm{MJ}$ in further investigation.

\section{Abbreviations}

MJ: Maoji Jiu; APH: N-acetylphenylhydrazine; CTX: Cyclophosphamide; WBC: White blood cell; RBC: Red blood cell; HGB: Hemoglobin; HCT: Hematocrit; PLT: Platelet; EPO: Erythropoietin; TGF- $\beta 1$ : Transforming growth factor-beta-1; M-CSF: Macrophage colony-stimulating factor; TCMs: Traditional Chinese medicines; FEJ: Fufang Ejiao Jiang; SOD: Superoxide dismutase; T-AOC: Total antioxidant activity; MDA: Malondialdehyde; ELISA: Enzyme-linked Immunosorbent assay; CG: Control group; MG: Model group; MJ$\mathrm{H}$ : High dose group of Maoji Jiu; MJ-M: Middle dose group of Maoji Jiu; MJ-L: Low dose group of Maoji Jiu; NS: Normal saline; H\&E: Hematoxylin-eosin; ABC-ELISA: Avidin-biotin peroxidase complex Enzyme- 
Linked Immunosorbent Assay; PCR: Reverse transcription polymerase chain reaction; cDNA: complementary DNA; RP: Red pulps; WP: White pulps; CR: Crypta.

\section{Declarations}

\section{Acknowledgements}

Not applicable.

\section{Ethics approval and consent to participate}

Female Sprague-Dawley rats were obtained from the Guangxi Medical University Laboratory Animal Centre (Nanning, China, License No. SCXK [Gui] 2014-0002). All procedures were carried in accordance with the ARRIVE (Animal Research: Reporting In Vivo Experiments) guidelines and approved by Ethics Committee of Guangxi Botanical Garden of Medicinal Plants (No. 20170301).

\section{Consent for publication}

Not applicable.

\section{Availability of data and materials}

All data generated and analyzed during this study are included in this article. The datasets used and/or analyzed during the current study are available from the corresponding author on reasonable request.

\section{Competing interests}

The authors declare that they have no competing interests.

\section{Funding}

This study was funded by the National Natural Science Foundation of China (81660724),Technology Development Program (GuangXi, China) grants (12118005-2-5), Medical and Health Appropriate Technology Development and Promotion Application project (GuangXi, China) (S2016 06).

\section{Authors' contributions}

ZGY and LL contributed equally to this work. All experiments were performed by FQZ, YLX, and CJZ. FQZ, YLX, JH, and ZGY wrote the manuscript. JPJ and JH were in charge of data analysis and technical graphics and helped revise the manuscript. ZGY and LL helped revise the manuscript. The authors read and approved the final manuscript.

\section{Author details}


${ }^{1}$ Guangxi Botanical Garden of Medicinal Plants, Nanning 530023, Guangxi, P.R. China. ${ }^{2}$ Department of Pharmacy, Guigang City People's Hospital, The Eighth Affiliated Hospital of Guangxi Medical University, Guigang 537100, Guangxi, P.R. China. ${ }^{3}$ Guangxi Institute of Chinese Medicine \& Pharmaceutical Science, Nanning 530022, Guangxi, P.R. China.

\section{References}

1. McLean E, Cogswell M, Egli I, Wojdyla D, de Benoist B. Worldwide Prevalence of anaemia, WHO vitamin and mineral nutrition information system, 1993-2005. Public Health Nutr. 2008;12:444-54.

2. Abeloff MD, Armitage JO Lichter AS. Clinical Oncology, second ed Voll. HartCourt, Churchill Living Stone 2001; pp:628-34.

3. Scheinberg P, Wu CO, Nunez O, Scheinberg P, Boss C, Sloand EM, Young NS. Treatment of severe aplastic anemia with a combination of horse antithymocyte globulin cyclosporine, with or without sirolimus: a prospective randomized study. Haematologica. 2009;94:348-54.

4. Marsh JC, Bacigalupo A, Schrezenmeier H, Tichelli A, Risitano AM, Passweg JR, Killick SB, Warren AJ, Foukaneli T, Aljurf M, Al-Zahrani HA, Höchsmann B, Schafhausen P, Roth A, Franzke A, Brummendorf TH, Dufour C, Oneto R, Sedgwick P, Barrois A, Kordasti S, Elebute MO, Mufti GJ, Socie G. Prospective study of rabbit antithymocyte globulin and cyclosporine for aplastic anemia from the EBMT severe aplastic anemia working party. Blood 2013;119:5391-6.

5. Li SJ, Lin H, Qu C, Tang YP, Shen J, Li WX, Yue SJ, Kai J, Shang GX, Zhu ZH, Zhang CB, Liu P, Yan H, Zhang L, Qian L, Qian DW, Duan JA. Urine and plasma metabonomics coupled with UHPLCQTOF/MS and multivariate data analysis on potential biomarkers in anemia and hematinic effects of Gui-Hong. J Ethnopharmacol. 2015;170:175-83.

6. Li WX, Tang YP, Guo JM, Shang EX, Qian YF, Wang LY, Zhang L, Liu P, Su SL, Qian DW, Duan JA. Comparative metabolomics analysis on hematopoietic functions of herb pair Gui-Xiong by ultra-highperformance liquid chromatography coupled to quadrupole time-of-flight mass spectrometry and pattern recognition approach. J Chromatogr A. 2014;1346:49-56.

7. Barreda DR, Haningron PC, Belosevic M. Regulation of myeloid development and function by colony stimulating factors. Dev Comp Immunol. 2004;28: 509-54.

8. Zhou J, Huang LP, Wu SF. Comparative study on three methods of blood-deficient mouse model. Lishizhen Med Meter Med Res. 2011;8:2007-8.

9. Zhang H, Wang HF, Liu Y, Huang LJ, Wang ZF, Li Y. The haematopoietic effect of Panax japonicus on blood deficiency model mice. J Ethnopharmacol. 2014;154:818-24.

10. Zeng FQ, Xu YL, Jiang SH, Zhang YY, Zhao CJ, Li L. Study on blood enriching effect of Centropus sinensis dried body alcohol extract on blood-deficiency model mice. J China Pharm. 2016;27:43735 .

11. Li PL, Sun HG, Hua YL, Ji P, Zhang L, Li JX, Wei Y. Metabolomics study of hematopoietic function of Angelica sinensis on blood deficiency mice. J Ethnopharmacol. 2015;166: 261-9. 
12. Li WX, Tang YP, Guo JM, Huang MY, Li W, Qian DW, Duan JA. Enriching blood effect comparison in three kinds of blood deficiency model after oral administration of drug pair of Angelicae Sinensis Radix and Chuangxiong Rhizom and each single herb. Chin J Chin Mater Med. 2011;36:1808-14.

13. Zhang JJ, Huang YF, Wang LL, Li W, Wang JX, Wang C, Qu SS. Comparative study on effects of blood enriching on mouse model of blood deficiency syndrome induced by compound method of bleeding, starved feeding exhausting of Paeoniae Radix Alba and Paeoniae Radix Rubra, paeoniflorin and albiflorin. Chin J Chin Mater Med. 2013;38:3358-62.

14. Liu MX, Tan HN, Zhang XK, Liu Z, Cheng YN, Wang DL, Wang FS. Hematopoietic effects and mechanisms of Fufang E'jiao Jiang on radiotherapy and chemotherapy-induced myelosuppressed mice. J Ethnopharmacol. 2014;152:575-84.

15. Wang M, Rang WQ, Zhang Q, Huo C, Ma ZC, Wang YG, Yan XZ, Gao Y. NMR-spectroscopy-based metabonomic approach to analysis of Siwutang, a novel prescription, treated blood deficiency in mice. Zhongguo Zhong Yao Za Zhi. 2010;35:630-4.

16. Huo C, Ma ZC, Wang M, Liu J, Yan XZ, Gao Y. NMR-spectroscopy-based metabonomic study on mouse model of blood deficiency syndrome induced by compound method of bleeding, starved feeding and exhausting and effect of siwutang. Zhongguo Zhong Yao Za Zhi. 2010;35:3194-8.

17. Wu CY, Chen R, Wang XS, Shen B, Yue W, Wu Q. Antioxidant and anti-fatigue activities of phenolic extract from the seed coat of Euryale ferox Salisb and identification of three phenolic compounds by LC-ESI-MS/MS. Molecules. 2013;18:11003-21.

18. Huang MY, Li WX, Tang YP, Guo JM, Shang EX, Wang YL, Qian DW, Duan JA. Comparative study on enriching blood effect of Fo-shou-san with different ethanol extraction. Chin J Clin Pharmacol Ther. 2011;16:841-6.

19. Xie ZL, Koyama T, Suzuki J, Fujii Y, Togashi H, Sawa H, Nagashima K. Coronary reperfusion following ischemia: different expression of bcl-2 and bax proteins, and cardiomyocyte apoptosis. Jpn Heart J. 2001;42:759-70.

20. McClintock DS, Santore MT, Lee VY, Brunelle J, Budinger GR, Zong WX, Thompson CB, Hay N, Chandel NS. Bcl-2 family members and functional electron transport chain regulate oxygen deprivation induced cell death. Mol Cell Biol. 2002;22:94-104.

21. Williams WV, VonFeldt JM, Rosenbaum H, Ugen KE, Weiner DB. Molecular cloning of a soluble form of the granulocyte-macrophage colony-stimulating factor receptor alpha chain from a myelomonocytic cell line. Expression, biologic activity, and preliminary analysis of transcript distribution. Arthritis Rheum. 1994;37: 1468-78.

22. Zhang H, Wang HF, Liu Y, Huang LJ, Wang ZF, Li Y. The haematopoietic effect of Panax japonicus on blood deficiency model mice. J Ethnopharmacol. 2014;154:818-24.

23. Wang SS, Zheng G, Tian SS, Zhang Y, Shen LJ, Pak Y, Shen Y, Qian J. Echinacoside improves hematopoietic function in 5-FU-induced myelosuppression mice. Life Sci. 2015;123:86-92.

24. Ripamonti V, Racca V, Calvo MG, Castiglioni P, Ferratini M. Angiotensin-converting enzyme inhibitors slow recovery from anaemia following cardiac surgery. Chest. 2016;130:79-84. 


\section{Tables}

Table 1 The changes of peripheral blood routine on blood deficiency model rats ( $\bar{x} \pm s, n=6)$.

\begin{tabular}{|llllll|}
\hline Group & $\mathrm{WBC} / \times 10^{9} / \mathrm{L}$ & $\mathrm{RBC} / \times 10^{12} / \mathrm{L}$ & $\mathrm{HGB} / \mathrm{g} \cdot \mathrm{L}^{-1}$ & $\mathrm{HCT} / \%$ & $\mathrm{PLT} / \times 10^{9} / \mathrm{L}$ \\
\hline CG & $9.15 \pm 1.00$ & $7.18 \pm 0.31$ & $135.67 \pm 6.12$ & $42.67 \pm 1.71$ & $929.0 \pm 140.04$ \\
\hline MG & $2.89 \pm 0.45^{\star}$ & $3.47 \pm 0.32^{*}$ & $89.17 \pm 10.82^{*}$ & $31.17 \pm 2.72^{*}$ & $646.0 \pm 62.66^{*}$ \\
\hline FEJ & $3.63 \pm 0.48^{\#}$ & $3.99 \pm 0.15^{\# \#}$ & $101.33 \pm 5.35^{\#}$ & $33.57 \pm 2.96$ & $810.5 \pm 159.79^{\#}$ \\
\hline MJ-H & $3.74 \pm 0.47^{\# \#}$ & $4.20 \pm 0.37^{\# \#}$ & $105.83 \pm 6.82^{\# \#}$ & $36.57 \pm 3.14^{\# \#}$ & $656.0 \pm 168.00$ \\
MJ-M & $3.55 \pm 0.54^{\#}$ & $3.95 \pm 0.34^{\#}$ & $100.17 \pm 4.22^{\#}$ & $35.33 \pm 2.18^{\#}$ & $679.8 \pm 125.57$ \\
\hline MJ-L & $3.16 \pm 0.88$ & $3.80 \pm 0.16^{\#}$ & $94.83 \pm 6.15$ & $33.33 \pm 1.53$ & $639.7 \pm 99.26$ \\
\hline
\end{tabular}

${ }^{*} P<0.01$ vs control group.

${ }^{\#} P<0.05$

${ }^{\# \#} P<0.01$ vs model group.

Table 2 The visceral index changes on blood deficiency model rats $(\overline{\mathrm{x}} \pm s, \mathrm{n}=6)$.

\begin{tabular}{|lllll|}
\hline Group & Liver index $(\mathrm{mg} / \mathrm{g})$ & Thymus index $(\mathrm{mg} / \mathrm{g})$ & Spleen index $(\mathrm{mg} / \mathrm{g})$ & Lung index $(\mathrm{mg} / \mathrm{g})$ \\
\hline CG & $25.97 \pm 1.30$ & $1.79 \pm 0.21$ & $2.35 \pm 0.12$ & $5.20 \pm 0.55$ \\
\hline MG & $32.52 \pm 3.01^{*}$ & $0.74 \pm 0.05^{*}$ & $5.41 \pm 0.47^{*}$ & $5.48 \pm 0.35$ \\
\hline FEJ & $29.23 \pm 1.65^{\#}$ & $0.87 \pm 0.12^{\#}$ & $5.13 \pm 1.05$ & $5.16 \pm 0.44$ \\
MJ-H & $29.75 \pm 0.52^{\#}$ & $0.94 \pm 0.12^{\# \#}$ & $4.84 \pm 0.40^{\#}$ & $5.35 \pm 0.23$ \\
MJ-M & $30.59 \pm 0.98$ & $0.87 \pm 0.09^{\#}$ & $4.91 \pm 0.25^{\#}$ & $5.33 \pm 0.39$ \\
MJ-L & $30.51 \pm 1.62$ & $0.81 \pm 0.18$ & $5.24 \pm 1.04$ & $5.32 \pm 0.69$ \\
\hline
\end{tabular}

${ }^{*} P<0.01$ vs control group.

${ }^{\#} P<0.05$

${ }^{\# \#} P<0.01$ vs model group.

Table 3 Effects of MJ on the activity of SOD, T-AOC and the level of MDA in livers of blood deficiency model rats $(\overline{\mathrm{x}} \pm s, \mathrm{n}=6)$. 


\begin{tabular}{|llll|}
\hline Group & $\begin{array}{l}\text { SOD } \\
\text { (U/mg prot) }\end{array}$ & $\begin{array}{l}\text { T-AOC } \\
(\mathrm{U} / \mathrm{mg} \text { prot })\end{array}$ & $\begin{array}{l}\text { MDA } \\
\text { (nmol/mg prot) }\end{array}$ \\
\hline CG & $197.50 \pm 7.92$ & $12.51 \pm 1.35$ & $9.45 \pm 1.05$ \\
\hline MG & $195.69 \pm 5.78$ & $8.68 \pm 1.50^{\star \star}$ & $15.44 \pm 2.11^{\star *}$ \\
\hline FEJ & $200.53 \pm 12.27$ & $10.54 \pm 0.85^{\#}$ & $12.98 \pm 1.02^{\#}$ \\
\hline MJ-H & $196.04 \pm 11.24$ & $12.47 \pm 1.37^{\# \#}$ & $12.40 \pm 1.08^{\# \#}$ \\
MJ-M & $195.43 \pm 6.47$ & $11.55 \pm 0.76^{\# \#}$ & $13.25 \pm 0.90^{\#}$ \\
\hline MJ-L & $194.50 \pm 15.42$ & $11.36 \pm 1.29^{\# \#}$ & $13.62 \pm 1.22$ \\
\hline
\end{tabular}

${ }^{\star} P<0.01$ vs control group.

${ }^{\#} P<0.05$

${ }^{\# \#} P<0.01$ vs model group.

\section{Figures}
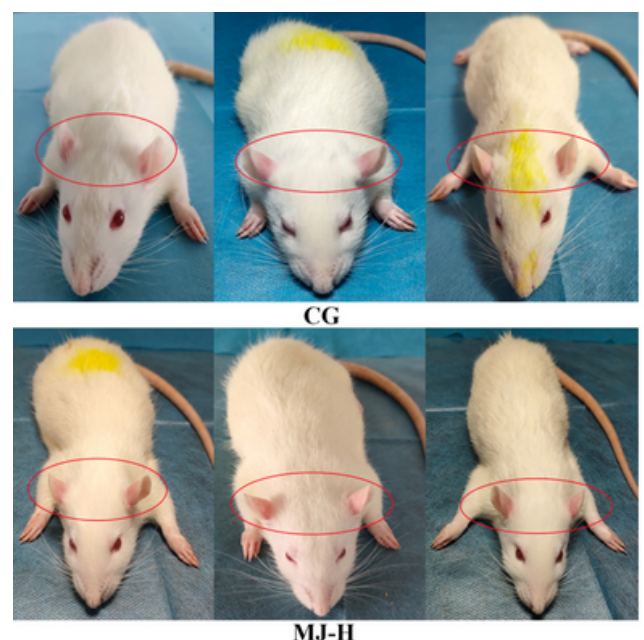

MJ-H
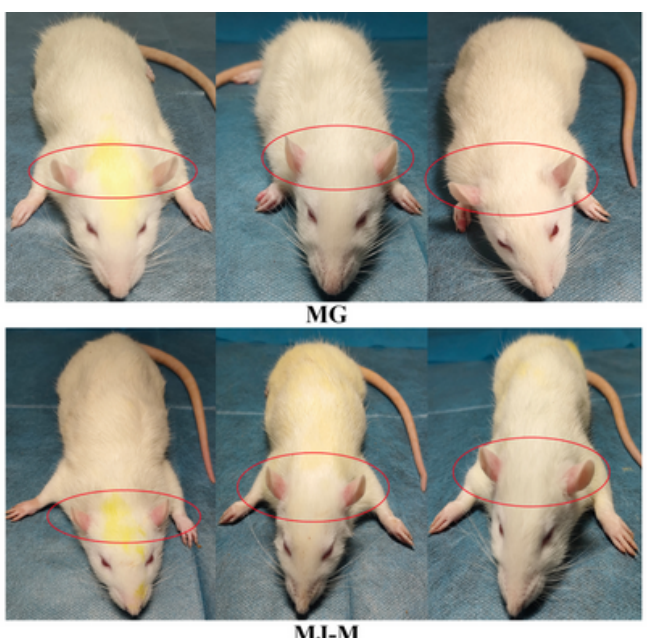

M.J-M

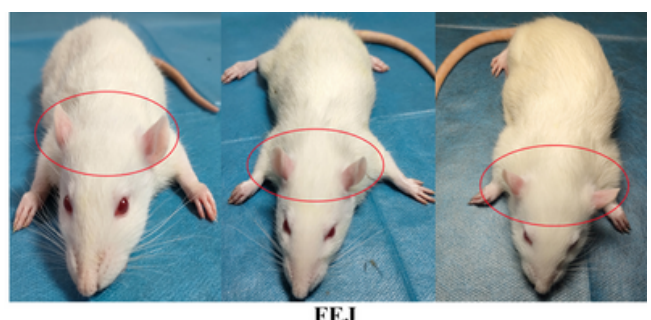

FEJ

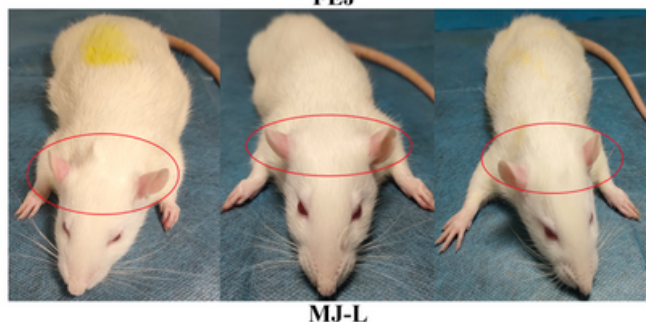

Figure 1

Change of MJ on appearance of the blood deficiency model rats. 


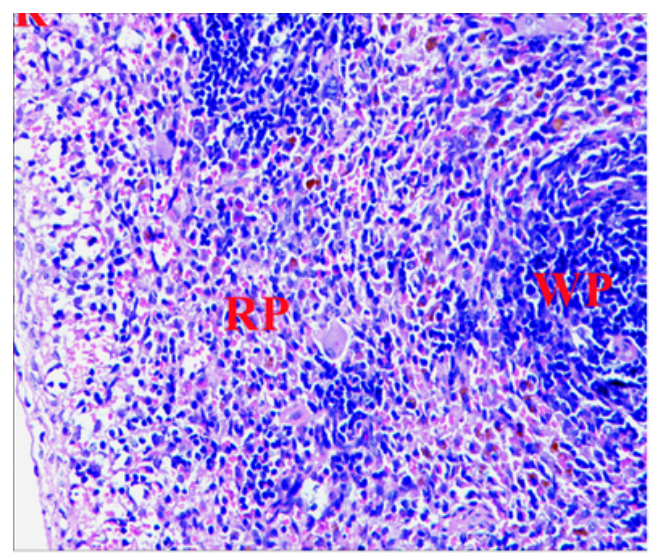

CG

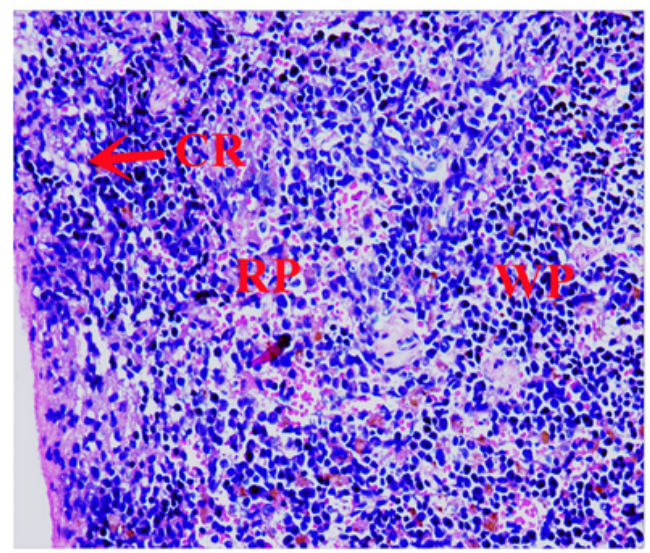

MJ-H

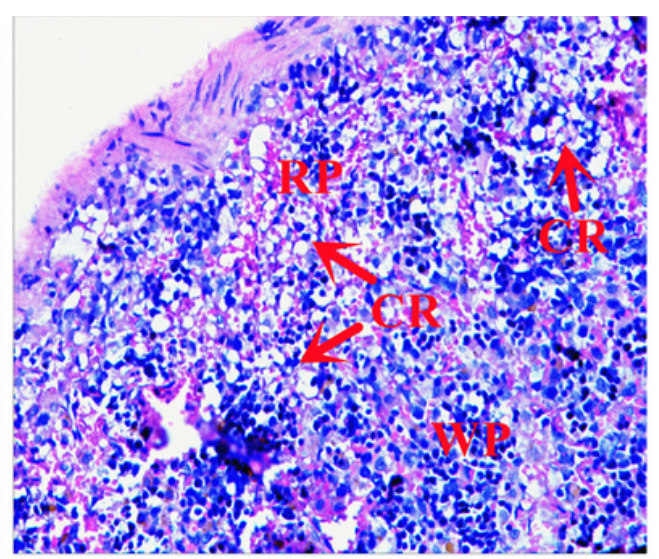

MG

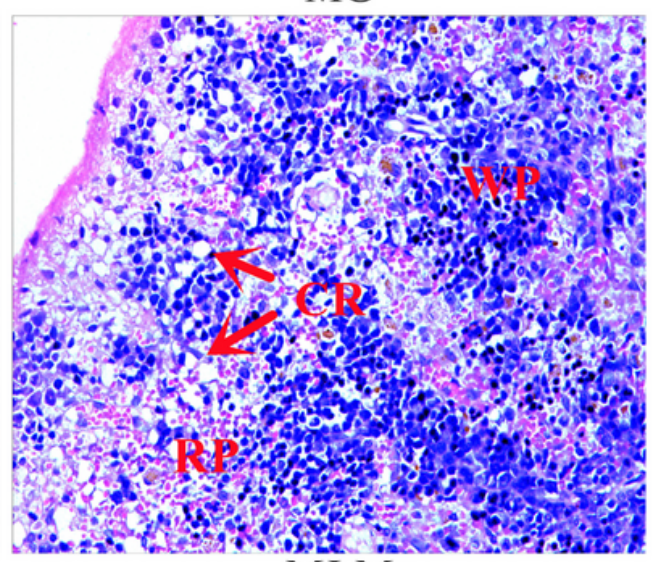

MJ-M

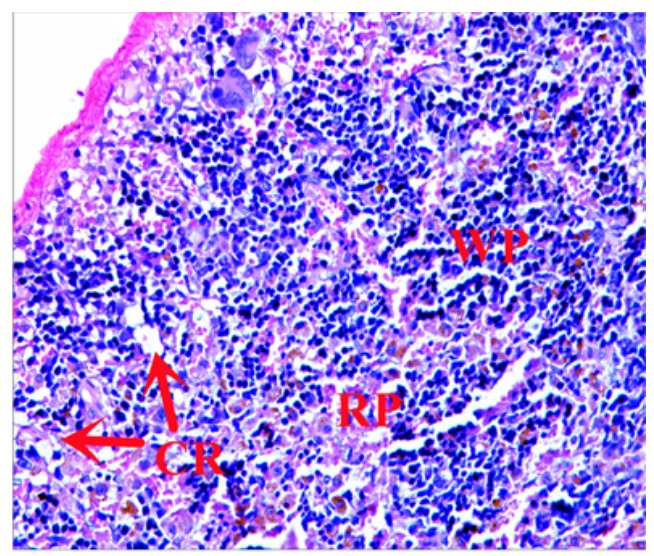

FEJ

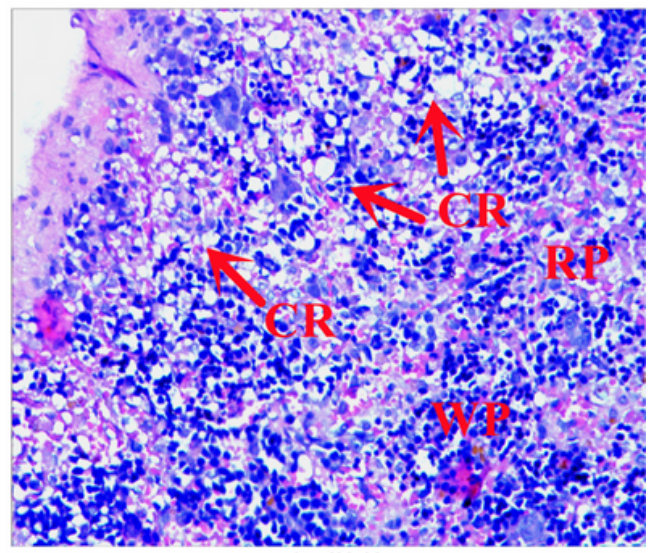

MJ-L

Figure 2

Effects of MJ on the histological structure in spleen tissues of blood deficiency model rats $(H E \times 200)$. 


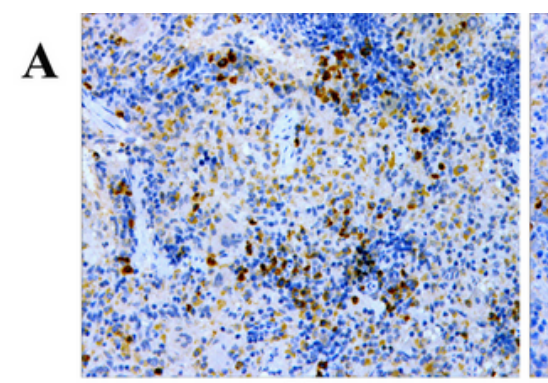

CG

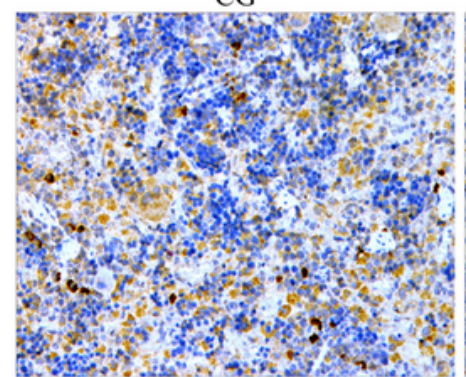

$$
\text { MJ-H }
$$

B
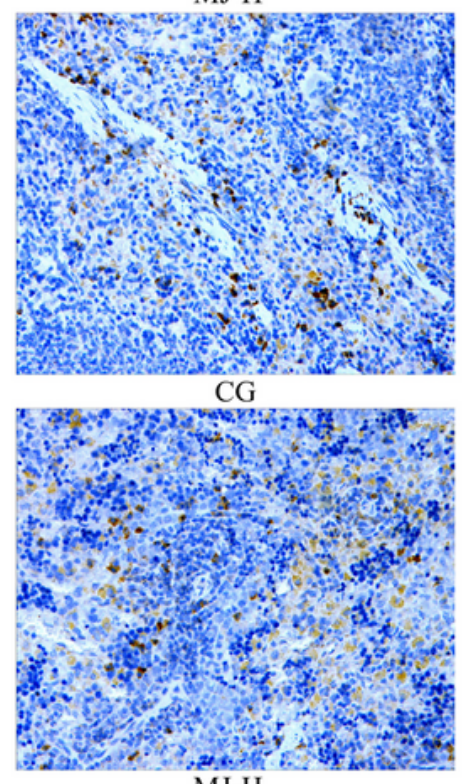

MJ-H

C

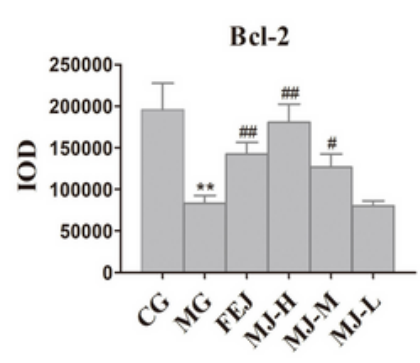

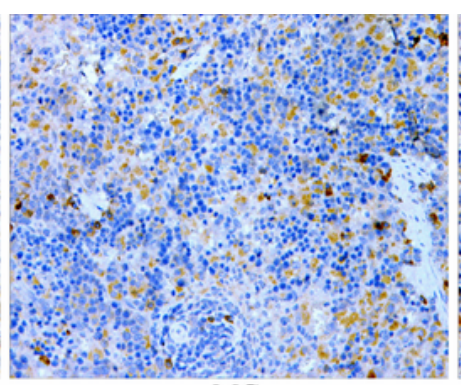

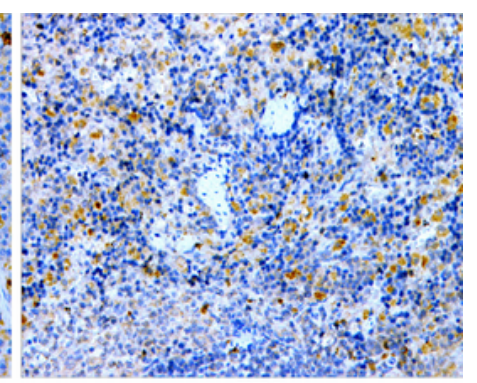

MG

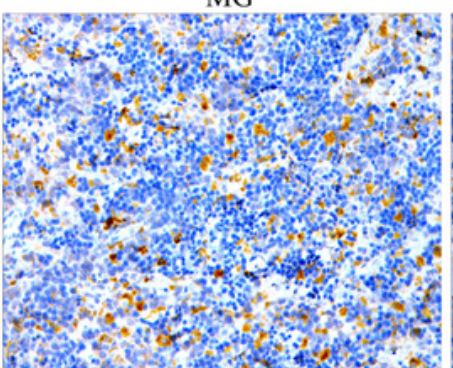

MJ-M

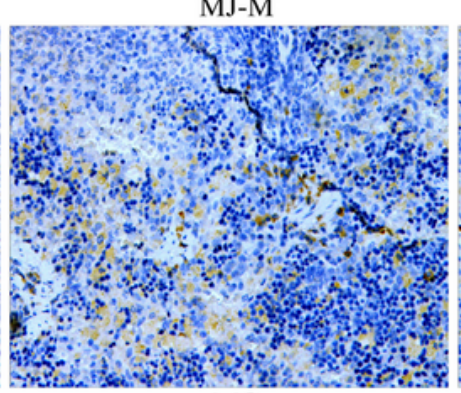

MG

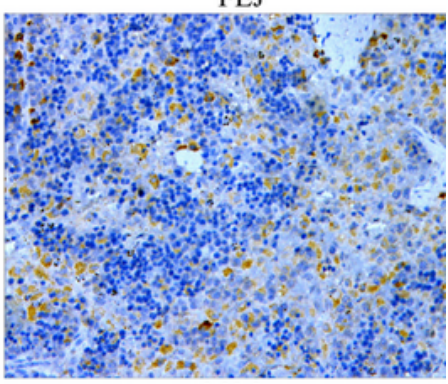

MJ-L

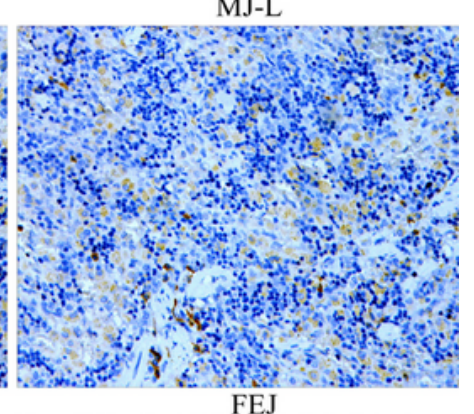

FEJ

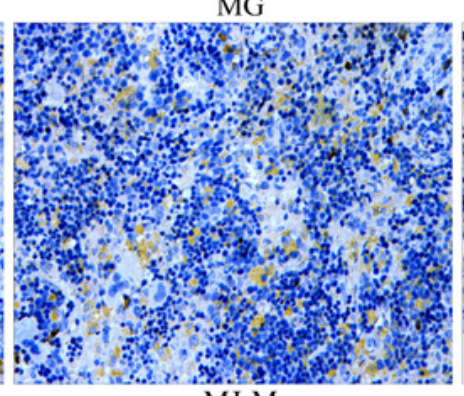

MJ-M

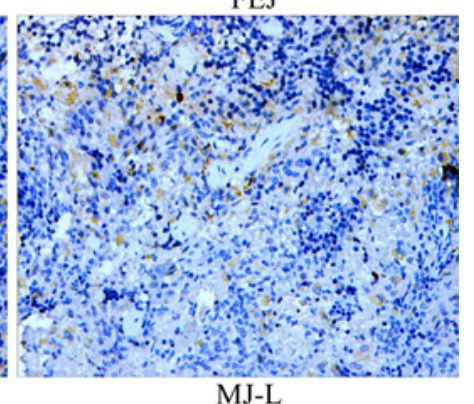

MJ-L

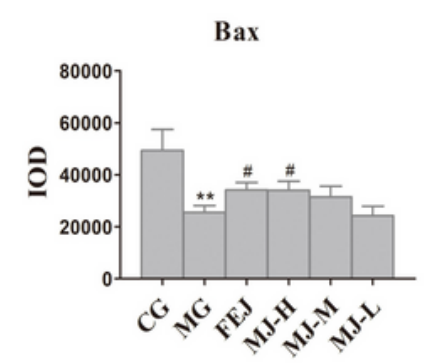

Bcl-2/Bax

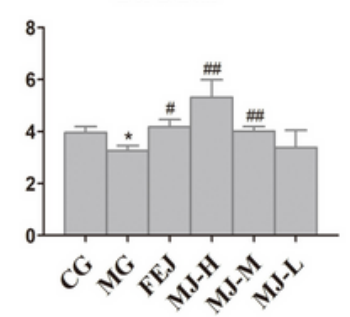

\section{Figure 3}

Effects of MJ on expression of Bcl-2 and Bax in spleen tissues of blood deficiency model rats.

(A)Representative photomicrographs of the SABC-stained histological slides of Bcl-2 in rat spleens (SABC $\times 200)$. (B)Representative photomicrographs of the SABC-stained histological slides of Bax in rat spleens $(S A B C \times 200)$. Using this staining method, the positive cell cytoplasm and cell nucleus are stained with 
claybank colour by SABC. (C)The means $\pm S D$ of the average absorbance values (IOD) of the splenic Bcl-2 and Bax proteins from the experimental animals are showed under the figure.

${ }^{*} \mathrm{P}<0.05,{ }^{\star *} \mathrm{P}<0.01$ vs control group; $\# \mathrm{P}<0.05, \# \# \mathrm{P}<0.01$ vs model group.

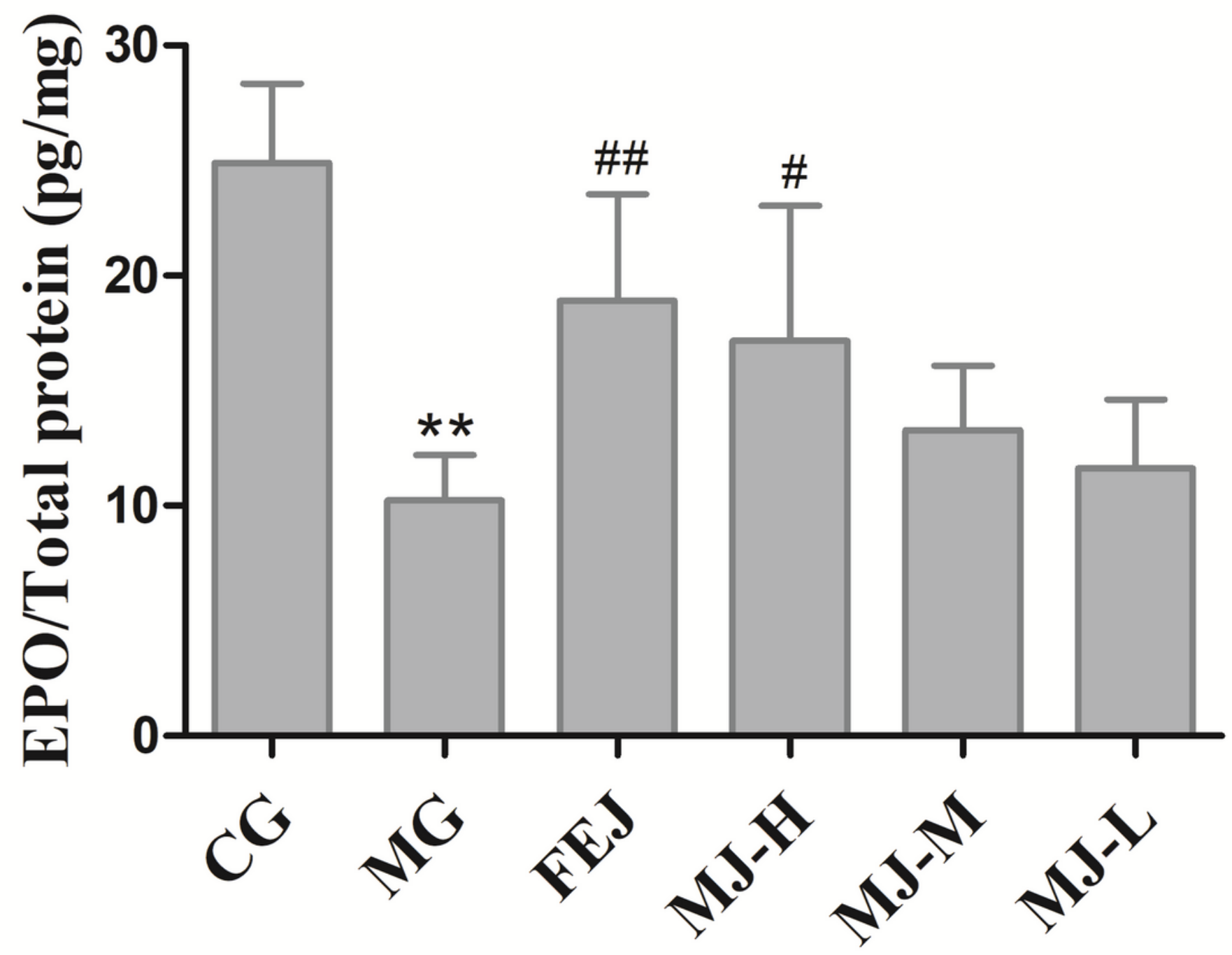

Figure 4

Effects of MJ on EPO level of spleen tissues of blood deficiency model rats.

${ }^{\star} P<0.05,{ }^{\star \star} P<0.01$ vs control group; $\# P<0.05, \# \# P<0.01$ vs model group. 


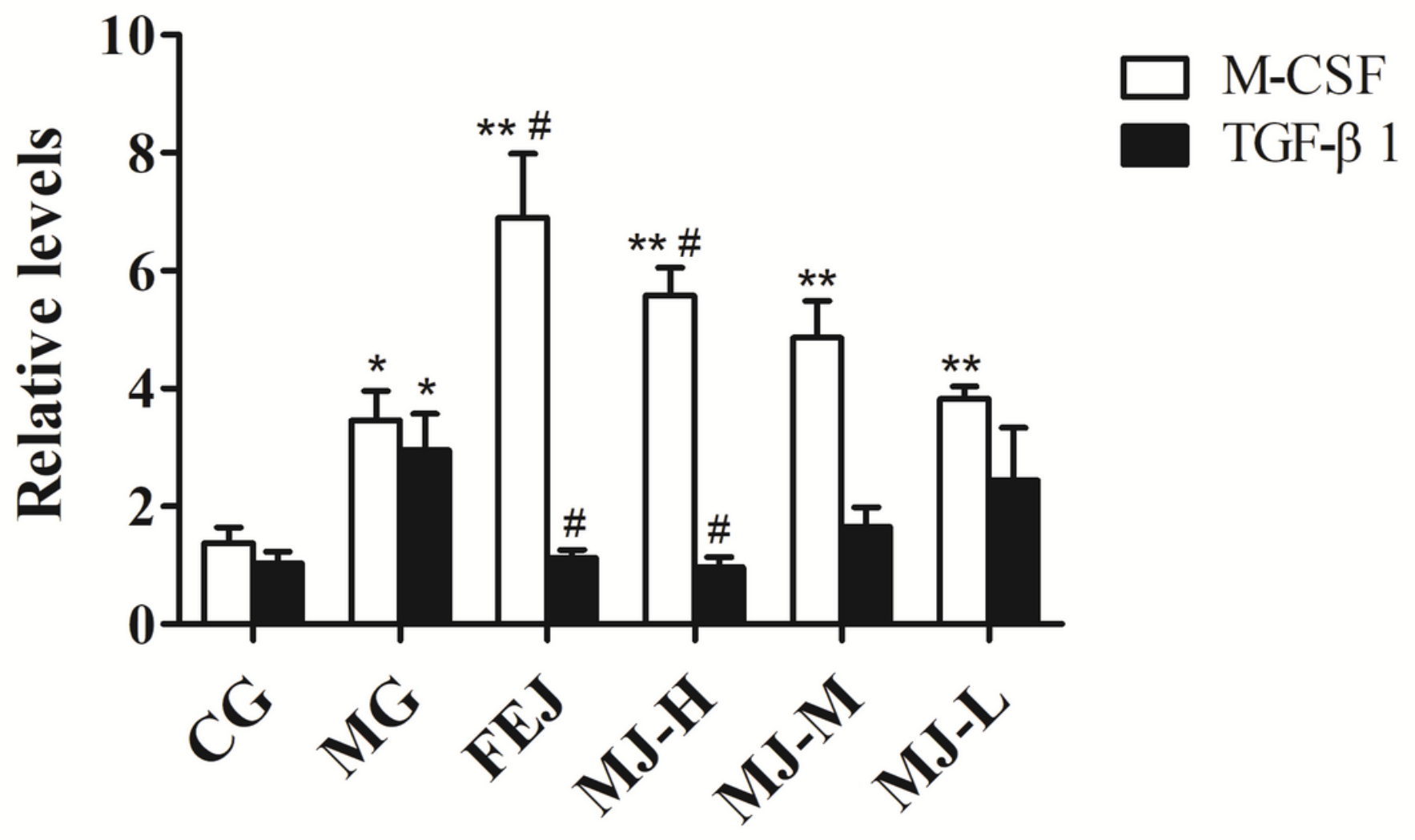

Figure 5

Effects of MJ on M-CSF and TGF- $\beta 1$ mRNA level of spleen tissues of blood deficiency model rats. **P<0.01 vs control group; $\# \mathrm{P}<0.05$ vs model group. 